\title{
100W, SINGLE MODE, SINGLE POLARIZATION, PICOSECOND, YTTERBIUM DOPED FIBRE MOPA FREQUENCY DOUBLED TO $530 \mathrm{~nm}$
}

\author{
K. K. Chen, Shaif-ul Alam, J. R. Hayes, D. Lin, A. Malinowski and D. J. Richardson \\ Optoelectronics Research Centre, University of Southampton, Southampton SO17 1BJ, UK
}

\section{Introduction}

High average power laser sources operating in the picosecond (ps) regime are useful for a wide range of applications including frequency-doubling and pumping of OPOs. Gain switching (GS) of laser diodes provides a practical and low cost method to generate ps pulses at $\mathrm{GHz}$ repetition rates and $\mathrm{mW}$ average power levels. Such devices represent excellent seeds for high power fiber MOPAs allowing power scaling to the $100 \mathrm{~W}$ regime and we recently reported average powers in excess of $300 \mathrm{~W}$ from a $1060 \mathrm{~nm}$ gainswitched FP laser seeded ytterbium doped fiber amplifier (YDFA) MOPA [1, 2]. However this system incorporated free space pump and signal coupling - greatly compromising the practicality of the system. Moreover, the output polarization was ill-defined limiting the utility of the system for many frequency conversion applications. Herein we present a fiberised, diode-seeded, YDFA MOPA system generating linearly polarized, diffraction-limited, 20ps pulses at repetition rates ranging from $113.5 \mathrm{MHz}$ to 908 $\mathrm{MHz}$ and at average output powers in excess of $100 \mathrm{~W}$. This system represents a considerable improvement in practicality and performance relative to previous high power, fiber-based ps pulse sources [3]. The output of the MOPA was launched into an LBO crystal to generate $45 \mathrm{~W}$ of green light.

\section{Experiment and Results}

Fig. 1 shows the experimental setup. A 1060nm FP laser diode in a high-speed fiber-pigtailed package was gain-switched using a pulsed drive current with an associated DC bias resulting in 20ps pulses with an average power of $1.3 \mathrm{~mW}$ at a repetition rate of $905 \mathrm{MHz}$. An inline EOM was used as a pulse picker to vary the pulse repetition rates. The excess loss of the EOM required us to use a three-stage YDFA MOPA chain. The first stage was a $4.5 \mathrm{~m}$ long core pumped YDFA followed by a $3.5 \mathrm{~m}$ long cladding pumped, single mode YDFA as the second stage. An in-line optical isolator was used to prevent ASE crosscoupling between the two stages.

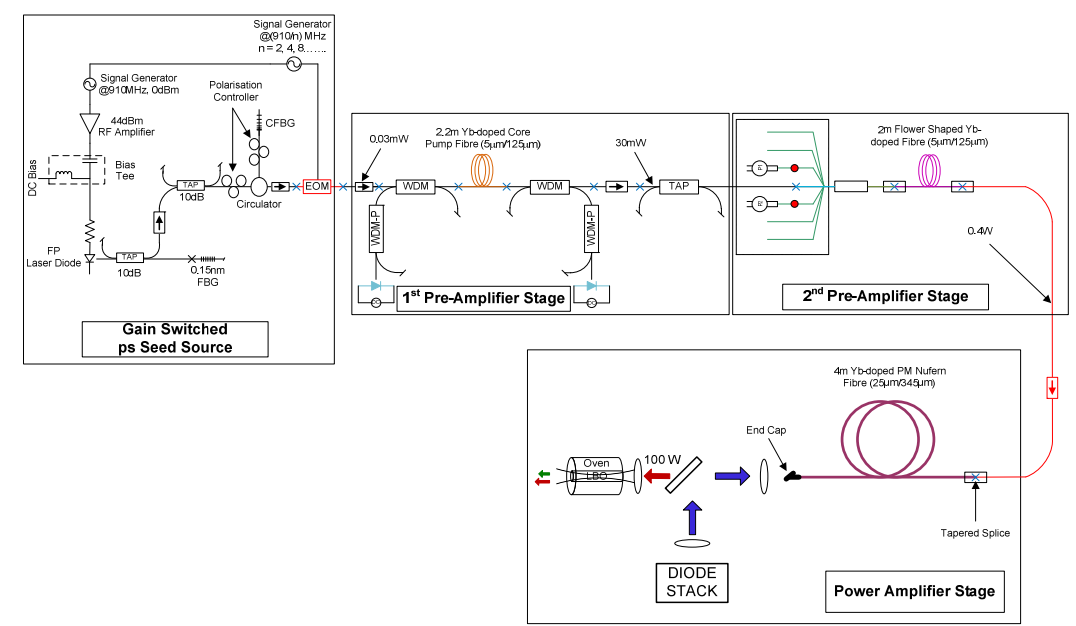

Fig. 1. Schematic diagram of the $\mathbf{Y b}^{3+}$-doped fiber MOPA.

The output of the second stage amplifier was coupled into a $4.5 \mathrm{~m}$ long, polarization maintaining (PM) power amplifier via a fast-axis blocking PM isolator. The amplifier was end-pumped using a wavelength stabilized, $975 \mathrm{~nm}$ diode stack. Output powers to $100 \mathrm{~W}$ were obtained at a slope efficiency of $77.6 \%$ as shown in Fig. 2. Beam quality $\left(\mathrm{M}^{2}\right)$ of the amplified signal output was measured to be $\sim 1.1-$ close to the diffraction limit. Measured polarisation extinction ratio (PER) was better than $19 \mathrm{~dB}$. The maximum extracted pulse energy was $0.88 \mu \mathrm{J}$ and corresponding peak power was $44 \mathrm{~kW}$ (at a repetition rate of 113.5 
MHz). A $20 \mathrm{GHz}$ PIN detector was used to measure the shape of both the seed and final output pulses. Fig.3 shows that no significant temporal distortion occurs during amplification. The corresponding full width at half maximum was measured to be $\sim 20$ ps using a Frequency Resolved Optical Gating (FROG) technique. The optical spectra of the amplified pulses were measured with an ANDO (AQ6317B) spectrum analyser Fig. 4 illustrates the SPM generated spectral broadening experienced by the amplified pulses inside the final stage amplifier with pulse repetition rate set to $227 \mathrm{MHz}$. The corresponding 3dB spectral bandwidth was measured at $0.9 \mathrm{~nm}$. The single polarisation output of the YDFA MOPA chain was focused into a $15 \mathrm{~mm}$ long LBO crystal using a $100 \mathrm{~mm}$ focal length lens. The diameter of the focused beam at the waist position was $70 \mu \mathrm{m}$, corresponding to a Rayleigh range of $12 \mathrm{~mm}$. A half-wave plate immediately before the focusing lens was used to rotate the polarization of the fundamental light to maximize the second harmonic signal. Average second harmonic power up to $45 \mathrm{~W}$ was achieved at an overall conversion efficiency of $45 \%$. Roll-off in SHG power, as shown in fig. 5, for output powers above $80 \mathrm{~W}$ was attributed to the observed to spectral broadening beyond the SHG crystal acceptance bandwidth. It should ultimately be possible to improve the conversion efficiency by carefully designing the final stage amplifier so as to better maintain the spectral integrity of the seed laser.

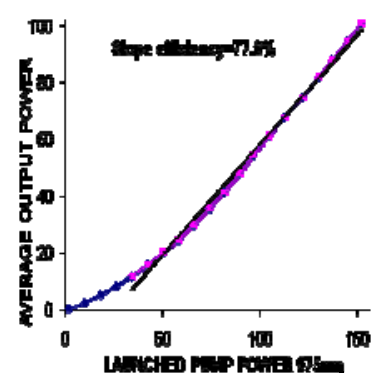

Fig. 2. Output vs pump power of the final stage amplifier

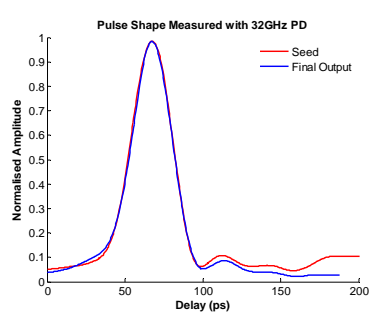

Fig. 3. Seed and amplified signals in the time domain

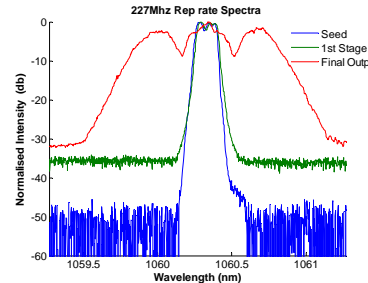

Fig. 4. Spectral plots corresponding to fig. 3

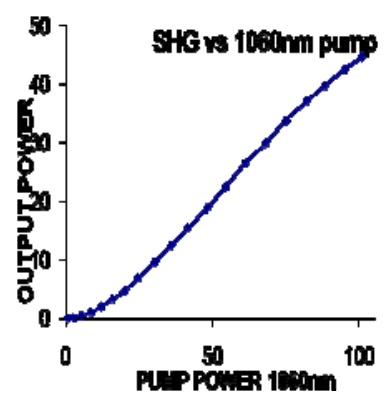

Fig. 5. SHG power as a function of fundamental signal.

\section{Conclusions}

We have successfully demonstrated a 100W, linearly polarized, near diffraction-limited, 20ps pulse source at $1.06 \mu \mathrm{m}$ based on a fiberised YDFA MOPA seeded by a GS-laser diode. Pulse energies to $0.85 \mu \mathrm{J}$ and peak powers $>40 \mathrm{~kW}$ were obtained when operating at a repetition rate of $113.5 \mathrm{MHz}$. Our experimental results show that further power scaling should be possible. Such high average power, singlepolarization, near diffraction-limited, ps sources are attractive for high power nonlinear frequency conversion and material processing applications. We have also generated $45 \mathrm{~W}$ of green light at $530 \mathrm{~nm}$ at an overall conversion efficiency of $45 \%$ by using an LBO crystal. Further power scaling is currently limited by the spectral integrity of the fundamental light. It should be possible to improve the conversion efficiency further by optimizing the final stage amplifier.

\section{Acknowledgements}

This work was funded in part by UK Technology Strategy Board grants LAMPS. The beam-corrected pump diode used in this work was produced by Heriot-Watt University/Power Photonics.

\section{References}

[1] P. Dupriez, A. Piper, A. Malinowski, J. K. Sahu, M. Ibsen, Y. Jeong, L. M. B. Hickey, M. N. Zervas, J. Nilsson, and D. J. Richardson, "321 W average power, $1 \mathrm{GHz}, 20$ ps, $1060 \mathrm{~nm}$ pulsed fiber MOPA source," Anaheim, CA, USA, 2005, p. 3 pp. Vol. 5.

[2] A. Piper, A. Malinowski, B. C. Thomsen, D. J. Richardson, L. M. B. Hickey, and M. N. Zervas, "11.1 W average power, 20 ps pulses at $1 \mathrm{GHz}$ repetition rate from a fiber-amplified gain-switched 1.06 $\mathrm{mm}$ Fabry-Perot laser diode," Baltimore, MD, USA, 2005, pp. 1141-3.

[3] J. L. Limpert, A; Gabler, T; Zellmer, H; Tünnermann, A; Unger, S; Jetschke, S; Müller, H -R, "High-averagepower picosecond Yb-doped fiber amplifier," Optics Letters, 26, 1849-1851 (2001). 